\title{
The systematic investigations of high-pressure polymorphs in shocked ordinary chondrites
}

M. Miyahara ${ }^{1}$, A. Yamaguchi ${ }^{2}$, M. Saitoh ${ }^{1}$, K. Fukimoto ${ }^{1}$, T. Sakai ${ }^{3}$, H. Ohfuji ${ }^{3}$, N. Tomioka ${ }^{4}$, Y. Kodama ${ }^{5}$ and E. Ohtani ${ }^{6}$

${ }^{1}$ Hiroshima Univ., Japan, ${ }^{2}$ NIPR, ${ }^{3}$ GRC, Ehime Univ., Japan, ${ }^{4}$ JAMSTEC, ${ }^{5}$ Marine Works Japan, ${ }^{6}$ Tohoku Univ., Japan

\section{Introduction}

A high-pressure polymorph occurring in a shocked meteorite can be a clue for clarifying an impact event occurred on a parent-body because we can constrain several parameters for the impact such as shock pressure and impact velocity using the high-pressure polymorph assemblage. In addition, the high-pressure polymorphs in the shocked meteorites are the analogs of the Earth's deep interior materials. Accordingly, many previous studies have worked on the high-pressure polymorphs in the shocked meteorites for several decades. The first discovery of the high-pressure polymorph is from one of L6 chondrite, and most previous investigations have focused on L6 because L6 is the most abundant among meteorites and we can easily find the high-pressure polymorphs. Tomioka and Miyahara (2017) [1] summarized the high-pressure polymorphs occurring in the shocked meteorites. Based on the review, many previous studies reported varied kinds of the high-pressure polymorphs from L6. On the other hand, few previous studies have worked on another petrologic type such as L5 and L4. The inventories of the high-pressure polymorphs in shocked H- and LL-group ordinary chondrites have been barely described so far. A parent-body of an ordinary chondrite is expected to have an onion shell-like structure. H-, L- and LL-group ordinary chondrites originate from individual parent-body. We have to clarify the inventories of high-pressure polymorphs included in all group ordinary chondrites to elucidate the destruction process of the ordinary chondrite parent-body. Accordingly, we initiated the systematic investigations of the highpressure polymorphs included in the shocked ordinary chondrites. In the first period of this project, we worked on L-group ordinary chondrites [2]. In the second and third periods, we worked on H- and LL-group ordinary chondrites. In this presentation, we will summarize all the descriptions.

\section{Experimental methods}

First, a shocked ordinary chondrite with melting texture was selected from Antarctica and non-Antarctica samples using an optical microscope for our purpose. Second, we took the whole area of the selected sample under BSE mode with a FEG-SEM. Subsequently, we observed the individual melting textures and phase transformation textures under higher magnification BSE image. Finally, we identified the high-pressure polymorphs by a laser micro-Raman spectroscopy.

\section{Results and discussion}

About sixty H-group, eighty L-group and fifty LL-group shocked ordinary chondrites with melting textures were systematically investigated following the procedures described above. There is the distinct difference on the mode of shockinduced melting between petrologic type 3-4 and type 5-6 in all group ordinary chondrites. In type 3-4 with melting textures, chondrules were slightly flattened. Melting occur at the boundaries between the flattened chondrules and surrounding matrices, which appear to be a melt-pocket. On the other hand, in type 5-6, melting occurs lineally; i.e., a shock-melt vein. There is also a clear difference in the occurrence rates of high-pressure polymorphs among H-, L- and LL-group. The species and assemblages of high-pressure polymorphs depended on the petrologic types. All L6 studied here includes high-pressure polymorphs (ringwoodite, wadsleyite, akimotoite, majorite, bridgmanite, majorite-pyrope solid-solution jadeite, lingunite and tuite). Not only the present investigation but also previous studies indicate that the occurrence ratio of high-pressure polymorphs in L6 is the highest among all ordinary chondrites. The number of species of high-pressure polymorphs in L6 is also the highest. In L5, although the occurrence ratio and a number of high-pressure polymorphs are lower than L6, most L5 include high-pressure polymorphs. Ringwoodite or ringwoodite + wadsleyite assemblage is the most dominant in L6 as the high-pressure polymorph of olivine, whereas in L5 sole wadsleyite is the most dominant. Na-pyroxene (mainly jadeite or omphacite), which occurs in plagioclase, is the most abundant high-pressure polymorph in L5. Both in L3 and L4, only Na-pyroxene occurs as a high-pressure polymorph. Few plagioclases occur in L3 and L4. Na-pyroxene occurs in the chondritic glass. LL6 includes many high-pressure polymorphs like L6. The number of species of high-pressure polymorphs is lower than L6. Wadsleyite and Na-pyroxene are the most abundant in LL6. In LL5, LL4 and LL3, only Na-pyroxene occurs. Few H6 include high-pressure polymorphs although distich melting textures were observed. In H5, H4 and H3, only a few of them include only Na-pyroxene. The number and assemblage of high-pressure polymorphs in shocked L- and LL-group are similar to each other, suggesting that both parentbodies have similar impact histories. On the other hand, the number and assemblage of high-pressure polymorphs in shocked $\mathrm{H}$ group are different from those of L- and LL-group, indicating that the impact history of H-group is different from those of Land LL-group. Acknowledgments: This research was supported by the NIPR through General Collaboration Project no. 26-31 and KP-307. References: [1] Tomioka N. and Miyahara M. Meteoritics and Planetary Science 52:2017-2039, 2017. [2] Miyahara M., Yamaguchi A., Ohtani E., Saitoh M. The Sixth Symposium on Polar Science, abstract, 2015. 